\title{
D-dimer for risk stratification and antithrombotic treatment management in acute coronary syndrome patients: a systematic review and metanalysis
}

Flavio Giuseppe Biccirè ${ }^{1,2}$, Alessio Farcomeni ${ }^{3}$, Carlo Gaudio ${ }^{1}$, Pasquale Pignatelli ${ }^{1}$, Gaetano Tanzilli ${ }^{1}$ and Daniele Pastori ${ }^{*}$ (D)

\begin{abstract}
Background: Data on the prognostic role of D-dimer in patients with acute coronary syndrome (ACS) are controversial. Our aim was to summarize current evidence on the association between D-dimer levels and short/ long-term poor prognosis of ACS patients. We also investigated the association between D-dimer and no-reflow phenomenon.
\end{abstract}

Methods: Systematic review and metanalysis of observational studies including ACS patients and reporting data on D-dimer levels. PubMed and SCOPUS databases were searched. Data were combined with hazard ratio (HR) and metanalysed. The principal endpoint was a composite of cardiovascular events (CVEs) including myocardial infarction, all-cause and cardiovascular mortality.

Results: Overall, 32 studies included in the systematic review with 28,869 patients. Of them, 6 studies investigated in-hospital and 26 studies long-term outcomes. Overall, 23 studies showed positive association of high D-dimer levels with CVEs. D-dimer levels predicted poor prognosis in all studies reporting in-hospital outcomes. Five studies satisfied inclusion criteria and were included in the metanalysis, with a total of 8616 patients. Median follow-up was 13.2 months with 626 CVEs. The pooled HR for D-dimer levels and CVEs was 1.264 (95\% Cl 1.134-1.409). Five out of 7 studies (4195 STEMI patients) investigating the association between D-dimer levels and no-reflow showed a positive correlation of D-dimer levels with no-reflow.

Conclusions: In patients with ACS, D-dimer was associated with higher in-hospital and short/long-term complications. D-dimer was also higher in patients with no-reflow phenomenon. The use of D-dimer may help to identify patients with residual thrombotic risk after ACS.

\footnotetext{
* Correspondence: daniele.pastori@uniroma1.it

'Department of Clinical Internal, Anesthesiological, and Cardiovascular

Sciences, Sapienza University of Rome, Viale del Policlinico 155, 00161 Rome, Italy

Full list of author information is available at the end of the article
}

(c) The Author(s). 2021, corrected publication 2022. Open Access This article is licensed under a Creative Commons Attribution 4.0 International License, which permits use, sharing, adaptation, distribution and reproduction in any medium or format, as long as you give appropriate credit to the original author(s) and the source, provide a link to the Creative Commons licence, and indicate if changes were made. The images or other third party material in this article are included in the article's Creative Commons licence, unless indicated otherwise in a credit line to the material. If material is not included in the article's Creative Commons licence and your intended use is not permitted by statutory regulation or exceeds the permitted use, you will need to obtain permission directly from the copyright holder. To view a copy of this licence, visit http://creativecommons.org/ licenses/by/4.0/. The Creative Commons Public Domain Dedication waiver (http://creativecommons.org/publicdomain/zero/1. 0/) applies to the data made available in this article, unless otherwise stated in a credit line to the data. 
Trial registration: The review protocol was registered in PROSPERO International Prospective Register of Systematic Reviews: CRD42021267233.

Keywords: D-dimer, Myocardial infarction, No-reflow phenomenon, Prognosis, Acute coronary syndrome

\section{Introduction}

Coronary artery disease (CAD) is the leading cause of death worldwide with acute myocardial infarction (MI) representing the clinical condition associated with the greatest morbidity and mortality, up to $30 \%$ of inhospital deaths $[1,2]$. Prompt reperfusion with percutaneous coronary intervention (PCI), advances in acute cardiovascular care and more effective medical therapy have improved the prognosis of patients with MI in the last years [3].

This increased survival revealed a previously unrecognized long-term risk of recurrent thrombotic events despite optimal medical therapy [2]. Indeed, almost one fifth of MI patients suffer from rehospitalization within 1 year and $10 \%$ from recurrent MI $[3,4]$.

There is therefore a growing need of a more accurate risk stratification of MI patients to identify those at higher risk for adverse events and worse prognosis [5].

Current guidelines of the American Heart Association and the European Society of Cardiology highlighted the lack of useful biomarkers to predict in-hospital complications and short/long term prognosis of patients with MI, recommending for this purpose only to measure troponin and brain natriuretic peptide (BNP) serum levels, with the latter associated with a low level of evidence $[6,7]$.

During last decades, several studies, mostly conducted in the 90's before the routine use of dual antiplatelet therapy, described the pivotal and prognostic role of coronary thrombosis and hypercoagulable state in the pathophysiology of MI, correlating increased coagulation activity with recurrent event and poor outcome after MI [8-10].

As a marker of rapid fibrin turnover and high thrombotic activation in both arterial and venous system, interest in D-dimer has grown over time, and its predictive role has been investigated in several acute and chronic cardiovascular care, [11]. In patients with MI, high circulating D-dimer levels have been correlated with recurrent MI and poor prognosis $[12,13]$ and early reduction in plasma concentration of D-dimer by Ximelagatran administration was associated with decreased risk of new cardiovascular events [14].

In addition, there are some data indicating that Ddimer may be higher in MI patients experiencing noreflow phenomenon [15], a severe condition in which high thrombotic burden interfere with complete restoration of myocardial blood supply after PCI, known to be correlated with adverse outcomes and poor prognosis [16].

Despite many studies published, conclusive data on the real usefulness and additional value of D-dimer in the management of patients with MI are lacking.

The aim of this systematic review and meta-analysis is to summarize current evidence on the potential prognostic role of D-dimer levels in CAD patients and its association with no-reflow phenomenon.

\section{Materials and methods Strategy search}

We conducted a systematic review of literature searching MEDLINE via PubMed and Scopus databases using a combination of the following keywords: "D-dimer" and "myocardial infarction" and "coronary artery disease" and "acute coronary syndrome". There was no time restriction and the last search was run on May 1, 2021. We included only journal articles in English language with full text available. We excluded case-control studies, case reports, editorials/comments, letters, review and meta-analysis, and experimental studies. Supplementary Fig. 1 report strategy searches which were performed according to the PRISMA guidelines. The systematic review was registered at PROSPERO (https://www.crd. york.ac.uk/PROSPERO) with registration number CRD42021267233.

\section{Study selection and quality assessment}

Two physicians (FGB, DP) independently screened the titles and abstracts of manuscripts identified through the database searches to identify studies potentially eligible for further assessment. A third physician (GT) reviewed eligible studies for appropriateness and completeness. The study selection was performed in multiple phases. In the first phase, potentially relevant studies were obtained by combined searches of electronic databases using the selected above-mentioned keywords and exclusion criteria. In the second phase, potentially eligible studies were reviewed to assess the appropriateness with the study question. Finally, the quality of pertinent studies included in the metanalysis was assessed by the Newcastle-Ottawa scale. Studies with a score $\geq 7$ were considered of good quality. Quality assessment is reported in Supplementary Table 1. 


\section{Endpoints}

The principal endpoint was a composite of cardiovascular events (CVEs) including MI, all-cause and cardiovascular mortality. The type of endpoint reported in each study is given in Table 1. Given the lack of high-quality studies, the association between D-dimer and no-reflow was not formally analysed but only systematically reviewed. Ethical approval was not required given the study type.

\section{Statistical analysis}

When available, hazard ratios (HRs) and odds ratios (ORs) were recorded from each study.

A separate meta-analysis was conducted for each endpoint, using a hierarchical Bayesian model which therefore would take into account heterogeneity among studies. This technique was chosen due to the limited number of studies included. Formally, each studyspecific effect was assumed to be distributed according to a log-Gaussian random variable, centered on a pooled effect. The variance on the log-scale was assumed to correspond to 1.25 times the squared standard error, as evaluated through confidence intervals reported in each study.

All analyses were conducted with $\mathrm{R}$ version 3.5.1, using the 'metafor and 'adaptMCMC' packages.

\section{Results}

\section{Characteristics of studies}

The association between D-dimer levels and clinical outcomes has been investigated in populations enrolled using different definitions of ischemic heart disease, such as CAD, ST segment elevation myocardial (STEMI), non-ST segment elevation myocardial infarction (NSTE $\mathrm{MI}$ ), acute MI and acute coronary syndrome (ACS). Characteristics of these studies have been separately described according to each definition (Table 1). Supplementary Table 2 and Supplementary Table 3 display the method used to dose D-dimer, cut-off levels and antithrombotic therapy across included studies.

\section{Cardiovascular events}

PRISMA flow diagram showing study search strategy about cardiovascular events is reported in Supplementary Fig. 1. After screening, 42 potentially eligible studies were identified and were considered for detailed analysis (Supplementary Fig. 1); 32 studies were included in the systematic review with 28,869 patients. Of these, 9 were retrospective, 20 prospective, 1 ambispective, 1 cross sectional study and 1 post-hoc analysis of a randomized clinical trial.

According to different inclusion criteria, 13 studies included STEMI, 3 NSTEMI, 7 MI, 9 CAD patients.

\section{ST segment elevation myocardial (STEMI)}

Overall, a sample of 7729 patients was analysed. The mean age ranged from 41 to 70.2 years and the proportion of women ranged from 8.45 to $54.7 \%$. Four studies investigated outcomes occurred during hospital stay, while 7 studies investigated long-term outcomes (Table 1).

Overall, 11 studies showed an association between high plasma D-dimer levels and CVEs in STEMI patients. In particular, D-dimer predicted worse prognosis in all studies considering in-hospital outcomes, and in 5 out of 7 studies with long-term follow-up (Table 1).

\section{Non-ST segment elevation myocardial infarction (NSTEMI)}

A total of 1949 NSTEMI patients, with a mean age that ranged from 57.2 to 67.4 years was included. The proportion of female patients ranged from 24.8 to $35.8 \%$. All studies investigated long-term prognosis. Overall, 1 out of 3 studies showed a positive association between high plasma D-dimer levels and CVEs incidence. However, one study showing no association between Ddimer and outcomes showed an association between Ddimer and NSTEMI severity, as assessed by Global Registry of Acute Coronary Events (GRACE) and thrombolysis in myocardial infarction (TIMI) risk scores, and included only mortality as endpoint; the other study had a short follow-up (6 months) and included also heart failure among cardiovascular outcomes (Table 1).

\section{Myocardial infarction}

Seven studies included a total of 12,320 patients with MI, with a mean age ranging from 62.8 to 74.2 years. The proportion of women ranged from 20 to $33.6 \%$ and 2 studies investigated in-hospital outcomes. Overall, 4 out of 7 studies consistently showed that D-dimer associated with CVEs (Table 1). Two studies also investigated the existence of a sex-based difference in the prognostic role of $\mathrm{D}$-dimer providing conflicting evidence. Indeed, the study by Wang et al. found that Ddimer predicted all-cause mortality in women but not men. In this study, women were significantly older than men ( 74 vs 61 years). The opposite was found in the THROMBO study, in which however the mean age was lower and the endpoint included a composited of nonfatal events and cardiovascular death (Table 1).

\section{Acute coronary syndrome (ACS) or CAD}

Nine studies included 6871 ACS/CAD patients with a mean age ranging from 57.2 to 71.1 years. The proportion of female patients ranged from 11.1 to $54.1 \%$. Overall, 8 out of 9 studies found that D-dimer was a predictive risk factor for CVEs in patients with ACS or CAD. In one study [45], the association between Ddimer and mortality was not confirmed at multivariable analysis; however, this study included a very 
Table 1 Clinical characteristics of studies investigating major adverse cardiac events and all-cause mortality included in the systematic review

\begin{tabular}{|c|c|c|c|c|c|c|c|c|c|}
\hline Author/ year & Setting & $N$ & Age (years) & $\begin{array}{l}\text { Women } \\
\text { (\%) }\end{array}$ & $\begin{array}{l}\text { Study } \\
\text { Design }\end{array}$ & $\begin{array}{l}\text { FU } \\
\text { (months) }\end{array}$ & Endpoints & D-dimer & Main findings \\
\hline \multicolumn{10}{|l|}{ STEMI } \\
\hline $\begin{array}{l}\text { Biccirè } 2021 \\
{[17]}\end{array}$ & STEMI & 132 & 64 & 19.1 & $\mathrm{R}$ & $\begin{array}{l}\text { In- } \\
\text { hospital }\end{array}$ & $\begin{array}{l}\text { Adverse events } \\
\text { (cardiogenic shock, } \\
\text { resuscitated cardiac } \\
\text { arrest and death). }\end{array}$ & $\begin{array}{l}\text { Events vs } \\
\text { control group: } \\
\text { log- } \\
\text { transformed D- } \\
\text { dimer } 6.8 \pm 1.1 \\
\text { vs } 6.3 \pm 0.8 \\
p=0.019\end{array}$ & $\begin{array}{l}\text { Patients experiencing } \\
\text { in-hospital adverse } \\
\text { events had higher } \\
\text { values of D-dimer com- } \\
\text { pared to those free } \\
\text { from events. }\end{array}$ \\
\hline $\begin{array}{l}\text { Huang } 2020 \\
{[18]}\end{array}$ & STEMI & 1165 & 63.5 & 17 & $\mathrm{R}$ & $\begin{array}{l}\text { In- } \\
\text { hospital }\end{array}$ & $\begin{array}{l}\text { CVEs including cardiac } \\
\text { death, non-fatal AMI, } \\
\text { revascularization, and } \\
\text { stroke. }\end{array}$ & $\begin{array}{l}\geq 800 \text { vs }<800 \\
\mathrm{ng} / \mathrm{mL}\end{array}$ & $\begin{array}{l}\text { Increased D-dimer level } \\
\text { predicted CVEs (OR } \\
8.408,95 \% \mathrm{Cl} 4.065- \\
17.392, P=0.001) \text {. D- } \\
\text { dimer AUC was 0.840 } \\
\text { (95\% Cl 0.769-0.911). } \\
\text { The best cut-off value } \\
\text { was } 640 \mathrm{ng} / \mathrm{mL} \text {. } \\
\text { In the subgroup with } \\
\text { no-reflow phenomenon, } \\
\text { increased D-dimer pre- } \\
\text { dicted CVEs (OR 8.114, } \\
95 \% \mathrm{Cl} 1.598-41.196, \\
p=0.012 \text { ) }\end{array}$ \\
\hline $\begin{array}{l}\text { Luo } 2020 \\
\text { [19] }\end{array}$ & STEMI & 400 & 62.5 & 21 & $\mathrm{R}$ & 12 & $\begin{array}{l}\text { CVEs (all-cause death, } \\
\text { TVR, MI, UA, HF, stroke } \\
\text { or TIA) }\end{array}$ & $\begin{array}{l}\text { Groups }(\mu \mathrm{g} / \mathrm{L}) \\
\text { 1: } 74.0 ; \\
\text { 2: } 146.0 ; \\
\text { 3: } 256.5 \\
\text { 4: } 576.0\end{array}$ & $\begin{array}{l}\text { The incidence of CVEs } \\
\text { and all-cause mortality } \\
\text { within } 30 \text { days ( } p< \\
0.001), 6 \text { months ( } p= \\
0.001) \text {, and } 1 \text { year ( } p= \\
0.001 \text { ) after } \mathrm{PCI} \text { in the } \\
\text { highest quartile of the } \\
\text { D-dimer groups were } \\
\text { higher than those in } \\
\text { the other } 3 \text { groups. }\end{array}$ \\
\hline $\begin{array}{l}\text { Qi Zhou } \\
2020 \text { [20] }\end{array}$ & STEMI & 872 & 63.7 & 19.8 & $\mathrm{R}$ & 29 & All-cause mortality & $\begin{array}{l}\text { Groups }(\mu \mathrm{g} / \\
\mathrm{mL}) \\
1: \leq 0.33 \\
\text { 2: } 0.33-0.64 \\
\text { 3: } 0.64-1.33 \\
4: \geq 1.33\end{array}$ & $\begin{array}{l}\text { Higher in-hospital HF } \\
(40.2 \text { vs } 10.2 \%, p< \\
0.0001), \text { malignant } \\
\text { arrhythmia }(14.2 \text { vs } \\
2.3 \%, p<0.0001) \text {, and } \\
\text { all-cause mortality ( } 5.9 \\
\text { vs } 0 \%, p<0.0001 \text { ) rates } \\
\text { were observed in Group } \\
4 . \\
84 \text { patients died. Group } \\
4 \text { was a predictor of all- } \\
\text { cause mortality (HR: } \\
2.53,95 \% \mathrm{Cl} 1.02-6.26 \text {, } \\
p=0.045) \text {. }\end{array}$ \\
\hline $\begin{array}{l}\operatorname{Lin} 2020 \\
{[21]}\end{array}$ & STEMI & 550 & 63.5 & 12.2 & $P$ & 16 & $\begin{array}{l}\text { Cl-AKI, in-hospital out- } \\
\text { comes and long-term } \\
\text { mortality and CVEs } \S\end{array}$ & $\begin{array}{l}\text { D-dimer } \\
\text { quartiles }(\mu \mathrm{g} / \\
\mathrm{mL}): \\
1:<0.38 \\
\text { 2: } 0.38-0.67 \\
\text { 3: } 0.68-1.03 \\
4:>1.03\end{array}$ & $\begin{array}{l}\text { D-dimer }>0.69 \mu \mathrm{g} / \mathrm{mL} \\
\text { was an independent } \\
\text { risk factor for long-term } \\
\text { mortality (HR: } 3.41[95 \% \\
\mathrm{Cl}, 1.4-8.03], p=0.005) \\
\text { and CVEs }\end{array}$ \\
\hline $\begin{array}{l}\text { Zhang } 2018 \\
\text { [22] }\end{array}$ & STEMI & 926 & 52.6 & 54.7 & $P$ & $\begin{array}{l}\text { In- } \\
\text { hospital }\end{array}$ & Mortality & $\begin{array}{l}383.1 \mathrm{ng} / \mathrm{mL} \pm \\
264.2\end{array}$ & $\begin{array}{l}\text { Patients without pre- } \\
\text { infarction angina with } \\
\text { high D-dimer level on } \\
\text { admission had signifi- } \\
\text { cantly increased in- } \\
\text { hospital mortality com- } \\
\text { pared to the other pa- } \\
\text { tients ( } p=0.041) \text {. }\end{array}$ \\
\hline Gao 2018 & STEMI & 822 & 62.5 & 46.1 & $P$ & 100 & Mortality & D-dimer 430.0 & Patients with high \\
\hline
\end{tabular}


Table 1 Clinical characteristics of studies investigating major adverse cardiac events and all-cause mortality included in the systematic review (Continued)

\begin{tabular}{|c|c|c|c|c|c|c|c|c|c|}
\hline Author/ year & Setting & $\mathbf{N}$ & Age (years) & $\begin{array}{l}\text { Women } \\
(\%)\end{array}$ & $\begin{array}{l}\text { Study } \\
\text { Design }\end{array}$ & $\begin{array}{l}\text { FU } \\
\text { (months) }\end{array}$ & Endpoints & D-dimer & Main findings \\
\hline$[23]$ & $\begin{array}{l}\text { with } \\
\text { T2DM }\end{array}$ & & & & & & & $\mathrm{ng} / \mathrm{mL} \pm 256.8$ & $\begin{array}{l}\text { plasma D-dimer level } \\
\text { on admission showed a } \\
\text { significantly shorter sur- } \\
\text { vival time ( } p<0.001 \text { in } \\
\text { the log-rank test). }\end{array}$ \\
\hline $\begin{array}{l}\text { Hansen } \\
2018[24]\end{array}$ & STEMI & 971 & 61 & 20 & $\begin{array}{l}\text { cross- } \\
\text { sectional } \\
\text { cohort } \\
\text { study }\end{array}$ & 55 & $\begin{array}{l}\text { Composite of all-cause } \\
\text { mortality, reinfarction, } \\
\text { stroke, unscheduled re- } \\
\text { vascularization, HF } \\
\text { rehospitalization } \\
\text { Secondary outcome } \\
\text { was total mortality }\end{array}$ & $\begin{array}{l}\text { Median D- } \\
\text { dimer } \\
456 \mathrm{ng} / \mathrm{mL}(\mathrm{IQR} \\
\text { 286-801). }\end{array}$ & $\begin{array}{l}\text { Adjusted OR for } \\
\text { composite endpoints } \\
\text { for D-dimer above } 456 \\
\text { ng/mL: } 1.179 \text { (95\% Cl, } \\
\text { 0.814-1.706 } p=0.384) \\
\text { Adjusted OR for total } \\
\text { mortality for D-dimer } \\
\text { above } 456 \mathrm{ng} / \mathrm{mL}: 2.01 \\
(95 \% \mathrm{Cl}, 1.06-3.83 ; p= \\
0.034)\end{array}$ \\
\hline $\begin{array}{l}\text { Sarli } 2015 \\
{[25]}\end{array}$ & STEMI & 266 & 64 & 38 & P & $\begin{array}{l}\text { in- } \\
\text { hospital }\end{array}$ & $\begin{array}{l}\text { CVEs: nonfatal MI, in- } \\
\text { stent thrombosis, and } \\
\text { in-hospital mortality } \\
\text { during hospitalization. }\end{array}$ & $\begin{array}{l}\text { D-dimer }(\mu \mathrm{g} / \mathrm{l}) \text { : } \\
686( \pm 236) \mathrm{vs} \\
418( \pm 164), p< \\
0.001\end{array}$ & $\begin{array}{l}\text { D-dimer level predicted } \\
\text { CVEs (OR: 1.002; 95\% Cl: } \\
\text { 1.000-1.004; } p=0.029) \text {. } \\
\text { Optimal cut-off value } \\
\text { was } 544 \mu \mathrm{g} / \mathrm{mL} \text { for } \\
\text { CVEs. }\end{array}$ \\
\hline $\begin{array}{l}\text { Erkol } 2014 \\
\text { [15] }\end{array}$ & STEMI & 569 & 56 & 16 & $A$ & 38 & $\begin{array}{l}\text { Mortality and CVEs } \\
\text { (death, non-fatal MI, } \\
\text { stroke, revasculariza- } \\
\text { tion, and advanced HF } \\
\text { at long-term follow-up) }\end{array}$ & $\begin{array}{l}\text { D-dimer overall } \\
0.40 \mathrm{mg} / \mathrm{L} \\
(0.20-0.87)\end{array}$ & $\begin{array}{l}\text { Univariable HR for long- } \\
\text { term mortality } 1.56 \\
(95 \% \mathrm{Cl}, 1.24-1.95, \mathrm{p}< \\
0.001) \text { and CVEs } 1.60 \\
(95 \% \mathrm{Cl}, 1.37-1.83, p< \\
0.001) \text {; not significant } \\
\text { association at multivari- } \\
\text { able analysis. }\end{array}$ \\
\hline $\begin{array}{l}\text { HORIZONS- } \\
\text { AMI } \\
\text { substudy } \\
2014[26]\end{array}$ & STEMI & 461 & $\begin{array}{l}\text { 1st: } 55.8 \\
\text { 2nd: } 61.0 \\
\text { 3rd: } 70.2\end{array}$ & 20.61 & $R$ & 36 & $\begin{array}{l}\text { CVEs (composite of all- } \\
\text { cause death, recurrent } \\
\text { MI, stroke, or TVR for } \\
\text { ischemia.) }\end{array}$ & $\begin{array}{l}\text { Tertiles }(\mu \mathrm{g} / \\
\mathrm{mL}): \\
1:<0.30(n= \\
215) \\
2: 0.30-0.71 \\
(n=161) \\
3: \geq 0.71(n= \\
85)\end{array}$ & $\begin{array}{l}\text { D-dimer levels } \\
\geq 0.71 \mu \mathrm{g} / \mathrm{mL} \text { on } \\
\text { admission predicted } \\
\text { CVEs (HR } 2.58 \text { [ } 95 \% \mathrm{Cl} \text {, } \\
1.44-4.63], p=0.0014 \text { ), } \\
\text { compared to the lowest } \\
\text { group. }\end{array}$ \\
\hline $\begin{array}{l}\text { Ozgur Akgul } \\
2013 \text { [27] }\end{array}$ & STEMI & 453 & 55.6 & 19.65 & P & 6 & Mortality & $\begin{array}{l}\text { High D-dimer } \\
\text { group: }> \\
0.72 \mu \mathrm{g} / \mathrm{mL} \text {; } \\
\text { Low D-dimer } \\
\text { group: lowest } \\
\text { two tertiles } \\
\text { ( } \leq 0.72 \mu \mathrm{g} / \mathrm{mL} \text { ). }\end{array}$ & $\begin{array}{l}\text { Highest tertile of D- } \\
\text { dimer associated with } \\
\text { in-hospital CV mortality } \\
\text { and 6-month all-cause } \\
\text { mortality ( } 7.2 \text { vs. } 0.6 \% \text {, } \\
p<0.001 \text { and } 13.9 \text { vs. } \\
2 \%, p<0.001 \text {, } \\
\text { respectively). } \\
\text { Fatal reinfarction, } \\
\text { advanced HF, and CVEs } \\
\text { were more frequent in } \\
\text { high D-dimer group } \\
\text { ( } p<0.001 \text { ). }\end{array}$ \\
\hline $\begin{array}{l}\text { Pineda } 2010 \\
\text { [28] }\end{array}$ & $\begin{array}{l}\text { AMI } \\
\text { STEMI } \\
(85.9 \%)\end{array}$ & 142 & 41 & 8.45 & $P$ & 36 & $\begin{array}{l}\text { Adverse CV events } \\
\text { included stroke, ACS, } \\
\text { CABG/PCl, } \\
\text { hospitalisation due to } \\
\text { congestive HF, CV and } \\
\text { global mortality. }\end{array}$ & $\begin{array}{l}\text { Event } 360.0 \mathrm{ng} / \\
\mathrm{mL} \text { vs no } \\
\text { event } 297.5 \mathrm{ng} / \\
\mathrm{mL}, p=0.314\end{array}$ & $\begin{array}{l}\text { No significant } \\
\text { differences in D-dimer } \\
\text { levels in the event } \\
\text { group. }\end{array}$ \\
\hline \multicolumn{10}{|l|}{ NSTEMI } \\
\hline Lu 2021 [29] & NSTEMI & 1357 & 65 & 31.4 & P & 12 & $\begin{array}{l}\text { Mortality and CVEs } \\
\text { including all-cause } \\
\text { death, hospital admis- } \\
\text { sion for UA and/or HF, } \\
\text { nonfatal recurrent MI }\end{array}$ & $\begin{array}{l}0.380 \mu \mathrm{g} / \mathrm{mL} \\
(0.27-0.65) \\
\text { Event group } \\
\text { (mortality at } 1 \\
\text { year): } 0.95\end{array}$ & $\begin{array}{l}\text { HR for D-dimer for 1- } \\
\text { year death and CVEs: } \\
2.12(95 \% C l, 1.50-2.99 \\
p<0.0001)\end{array}$ \\
\hline
\end{tabular}


Table 1 Clinical characteristics of studies investigating major adverse cardiac events and all-cause mortality included in the systematic review (Continued)

\begin{tabular}{|c|c|c|c|c|c|c|c|c|c|}
\hline Author/ year & Setting & $N$ & Age (years) & $\begin{array}{l}\text { Women } \\
(\%)\end{array}$ & $\begin{array}{l}\text { Study } \\
\text { Design }\end{array}$ & $\begin{array}{l}\text { FU } \\
\text { (months) }\end{array}$ & Endpoints & D-dimer & Main findings \\
\hline & & & & & & & and stroke) & $\begin{array}{l}(0.50,2.00) \\
\text { Control group: } \\
0.37(0.26,0.60)\end{array}$ & \\
\hline $\begin{array}{l}\text { Hulusi } \\
\text { Satilmisoglu } \\
2017[30]\end{array}$ & NSTEMI & 234 & 57.2 & 24.8 & $\mathrm{R}$ & 14 & Mortality & $\begin{array}{l}\text { Non-survivors: } \\
1568 \pm 1489 \\
\text { ng/mL } \\
\text { Survivors: } \\
632 \pm 995 \text { ng/ } \\
\mathrm{mL}\end{array}$ & $\begin{array}{l}\text { D-dimer correlated with } \\
\text { GRACE ( } r=0.215, p= \\
0.01) \text { and TIMI scores } \\
(r=0.253, p<0.001) \text {. } \\
\text { Higher levels recorded } \\
\text { for D-dimer assay ( } p= \\
0.003) \text { in non-survivors. } \\
\text { At multivariate analysis, } \\
\text { D-dimer assay no sig- } \\
\text { nificant predictor of in- } \\
\text { creased mortality risk. }\end{array}$ \\
\hline $\begin{array}{l}\text { Tello- } \\
\text { Montoliu } \\
2007[31]\end{array}$ & NSTEMI & 358 & 67.4 & 35.8 & $\mathrm{R}$ & 6 & $\begin{array}{l}\text { Death, new ACS, } \\
\text { revascularization, and } \\
\text { HF }\end{array}$ & $\begin{array}{l}\text { Overall D- } \\
\text { dimer level: } \\
340 \text { (211-615) } \\
\text { ng/mL }\end{array}$ & $\begin{array}{l}\text { Admission D-dimer } \\
\text { levels did not predict } \\
\text { events [HR: } 1.26(0.79- \\
\text { 2.02), } p=0.337) \text {. }\end{array}$ \\
\hline \multicolumn{10}{|l|}{ AMI } \\
\hline Fu 2020 [32] & $\begin{array}{l}\text { AMI with } \\
\text { ESRD }\end{array}$ & 113 & 69.2 & 33.6 & $\mathrm{R}$ & $\begin{array}{l}\text { In- } \\
\text { hospital }\end{array}$ & Mortality & $\begin{array}{l}\text { Mortality: } 3.2 \\
\text { mg/L } \\
\text { Survival: } 1.1 \\
\text { mg/L } \\
p=0.023\end{array}$ & $\begin{array}{l}\text { D-dimer } \geq 2.4 \mathrm{mg} / \mathrm{L} \\
\text { predicted in-hospital } \\
\text { mortality (OR } 2.771 \\
{[95 \% \mathrm{Cl}, 1.017-8.947]} \\
\mathrm{p}<0.001 \text { ) }\end{array}$ \\
\hline $\begin{array}{l}\text { Wang } 2020 \\
\text { [33] }\end{array}$ & AMI & 197 & $\begin{array}{l}\text { Male } 61.8 \\
\text { Female } 74.2\end{array}$ & 20 & P & 6 & $\begin{array}{l}\text { All-cause mortality (in- } \\
\text { and out-of-hospital } \\
\text { deaths) or readmission. }\end{array}$ & $\begin{array}{l}\text { Male D-dimer } \\
(\mathrm{mg} / \mathrm{L}) 0.4 \mathrm{vs} \\
1.0 P<0.001 \\
\text { Female D- } \\
\text { dimer }(\mathrm{mg} / \mathrm{L}) \\
0.4 \text { vs } 0.6 \\
p=0.015\end{array}$ & $\begin{array}{l}\text { HR for continuous D- } \\
\text { dimer in women 2.029 } \\
(95 \% \mathrm{Cl}, 1.403-2.933 ; \\
\mathrm{p}<0.001) \text {. D-dimer } \\
\geq 0.43 \mathrm{mg} / \mathrm{L} \text { as an inde- } \\
\text { pendent predictor of } \\
\text { poor prognosis in fe- } \\
\text { male AMl patients. }\end{array}$ \\
\hline $\begin{array}{l}\text { Zhang } 2020 \\
\text { [34] }\end{array}$ & $\mathrm{AMI}$ & 4495 & 62 & 31.03 & P & 24 & All-cause mortality & $\begin{array}{l}<145 \mathrm{ng} / \mathrm{mL} \\
\geq 145 \mathrm{ng} / \mathrm{mL}\end{array}$ & $\begin{array}{l}\text { Elevated D-dimer was } \\
\text { associated with mortal- } \\
\text { ity (univariable HR 1.20, } \\
95 \% \mathrm{Cl}, 1.04-1.37, \mathrm{p}= \\
0.01 \text { ) and in the pa- } \\
\text { tients in different } \\
\text { groups (HFpEF, HFrEF, } \\
\text { non-HF). }\end{array}$ \\
\hline Yu 2019 [35] & AMl & 5923 & 62.2 & 30.5 & P & $\begin{array}{l}\text { In- } \\
\text { hospital }\end{array}$ & Mortality & $\begin{array}{l}\text { D-dimer tertiles } \\
\text { (ng/mL): } \\
\text { Low: } \leq 88 ; \\
\text { Intermediate: } \\
\text { 89-179; High: } \\
>179 .\end{array}$ & $\begin{array}{l}\text { After multivariable } \\
\text { adjustment, D-dimer } \\
\text { significantly predicted } \\
\text { in-hospital mortality (OR } \\
1.060[95 \% \mathrm{Cl}, 1.026- \\
\text { 1.094], } p<0.001) . \\
\text { D-dimer levels } \\
\text { significantly improved } \\
\text { the prognostic } \\
\text { performance of GRACE } \\
\text { score (C-statistic: } p= \\
2.269, p=0.023 ; \text { IDI: } \\
0.016, p=0.032 ; \text { no- } \\
\text { reflowl: } 0.291, p=0.035) \text {. }\end{array}$ \\
\hline $\begin{array}{l}\text { REBUS study } \\
2017 \text { [36] }\end{array}$ & AMl & 412 & 67 & 22.3 & P & 24 & $\begin{array}{l}\text { Composite endpoint } \\
\text { (all-cause death, Ml, } \\
\text { congestive HF, or all- } \\
\text { cause stroke) }\end{array}$ & $\begin{array}{l}\text { Median D- } \\
\text { dimer was } \\
677 \mu \mathrm{g} / \mathrm{L} \text { at } \\
\text { inclusion }\end{array}$ & $\begin{array}{l}\text { D-dimer was not } \\
\text { associated with the } \\
\text { composite endpoint } \\
\text { (HR } 1.22 \text { [95\% Cl 0.99- } \\
1.51], p=0.06 \text {, for one } \\
\text { SD increase). }\end{array}$ \\
\hline Smid 2011 & AMl & 135 & 61 & 26 & $P$ & 12 & CV death, recurrent $\mathrm{MI}$ & On admission & D-dimer on admission \\
\hline
\end{tabular}


Table 1 Clinical characteristics of studies investigating major adverse cardiac events and all-cause mortality included in the systematic review (Continued)

\begin{tabular}{|c|c|c|c|c|c|c|c|c|c|}
\hline Author/ year & Setting & $N$ & Age (years) & $\begin{array}{l}\text { Women } \\
\text { (\%) }\end{array}$ & $\begin{array}{l}\text { Study } \\
\text { Design }\end{array}$ & $\begin{array}{l}\text { FU } \\
\text { (months) }\end{array}$ & Endpoints & D-dimer & Main findings \\
\hline$[37]$ & & & & & & & $\begin{array}{l}\text { a second } \mathrm{PCI} \text { or } \mathrm{CABG} \\
\text { and ischemic stroke. }\end{array}$ & $\begin{array}{l}\text { D-dimer: } 370 \\
(260-718) \mathrm{ng} / \\
\mathrm{mL}\end{array}$ & $\begin{array}{l}\text { was higher in patients } \\
\text { with recurrent } \\
\text { thrombotic CV event } \\
\text { (medians } 550 \text { vs. } 365 \\
\text { ng/mL, p }=0.06 \text { ) } \\
\text { OR for D-dimer against } \\
\text { endpoints: } 2.9 \text { ( } 95 \% \mathrm{Cl} \\
\text { 0.9-8.8) }\end{array}$ \\
\hline $\begin{array}{l}\text { THROMBO } \\
\text { study } 2000 \\
{[38]}\end{array}$ & AMI & 1045 & $\begin{array}{l}\text { Male } 58 \\
\text { Female } 62\end{array}$ & 24.3 & P & 26 & $\begin{array}{l}\text { Recurrent cardiac } \\
\text { events (nonfatal } \\
\text { reinfarction or cardiac } \\
\text { death) }\end{array}$ & $\begin{array}{l}\text { D-dimer mean } \\
\text { in men } 508 \pm \\
690 \mathrm{ng} / \mathrm{mL} \text { vs } \\
\text { women } 564 \pm \\
430 \mathrm{ng} / \mathrm{mL}\end{array}$ & $\begin{array}{l}\text { D-dimer had prognostic } \\
\text { value in men (HR 2.35, } \\
95 \% \mathrm{Cl} 1.27-4.35], p= \\
0.006) \text { but not in } \\
\text { women (HR 1.58, 95\%Cl } \\
0.59-4.22, p=0.360) \text {. }\end{array}$ \\
\hline
\end{tabular}

\section{ACS/CAD}

\begin{tabular}{|c|c|c|c|c|c|c|c|c|c|}
\hline $\begin{array}{l}\text { Chen } 2018 \\
\text { [39] }\end{array}$ & $\begin{array}{l}\text { CAD } \\
(76.9 \% \\
\text { AMI) }\end{array}$ & 238 & 64.4 & 21.1 & P & 24 & $\begin{array}{l}\text { All-cause mortality and } \\
\text { CVEs (cardiac death } \\
\text { and nonfatal } \\
\text { outcomes: recurrent } \\
\text { MI, TVR or re-admission } \\
\text { due to advanced HF). }\end{array}$ & $\begin{array}{l}\text { Mean D-dimer: } \\
0.7 \pm 1.1 \mathrm{mg} / \mathrm{L}\end{array}$ & $\begin{array}{l}\text { OR for long-term CVEs: } \\
1.526 \text { (95\% Cl, 1.174- } \\
1.983), p=0.002 . \\
\text { D-dimer in multivariate } \\
\text { Cox regression of CVEs: } \\
1.420(1.069-3.014), p= \\
0.046\end{array}$ \\
\hline $\begin{array}{l}\text { Kosaki } 2018 \\
{[40]}\end{array}$ & $\begin{array}{l}\text { ACS } \\
(76.3 \% \\
\text { AMI) }\end{array}$ & 400 & 71.1 & 27.2 & P & 27 & $\begin{array}{l}\text { CVEs (all-cause } \\
\text { mortality, recurrent MI, } \\
\text { unplanned repeat } \\
\text { revascularization, } \\
\text { surgical } \\
\text { revascularization, fatal } \\
\text { arrhythmia, admission } \\
\text { for HF, and stroke. }\end{array}$ & $\begin{array}{l}\text { Patients } \\
\text { without CVEs: } \\
1.67 \mathrm{mg} / \mathrm{mL} \pm \\
2.49 \\
\text { Patients with } \\
\text { CVEs: } 2.11 \mathrm{mg} / \\
\mathrm{mL} \pm 2.72 \\
p=0.0003\end{array}$ & $\begin{array}{l}\text { Univariate analysis for } \\
\text { D-dimer } \geq 0.84 \mathrm{mg} / \mathrm{mL} \\
\text { predicting CVEs: OR } \\
2.49(95 \% \mathrm{Cl} 1.54-4.11) \\
p=0.0001\end{array}$ \\
\hline $\begin{array}{l}\text { ATLAS ACS- } \\
\text { TIMI46 Trial } \\
\text { Substudy } \\
2018 \text { [41] }\end{array}$ & $\begin{array}{l}\text { ACS } \\
(73.9 \% \\
\text { AMI) }\end{array}$ & 1834 & $\begin{array}{l}\text { Placebo } 57.9 \\
\text { Rivaroxaban } \\
57.2\end{array}$ & 23.2 & $\begin{array}{l}\text { Post-hoc } \\
\text { RCT }\end{array}$ & 6 & $\begin{array}{l}\text { Composite endpoint of } \\
\text { CV death, myocardial } \\
\text { infarction, or stroke }\end{array}$ & $\begin{array}{l}\text { Baseline } D \text { - } \\
\text { dimer }(\mu \mathrm{g} / \mathrm{mL}) \\
\text { Placebo } 0.39 \\
(0.24-0.73) \text { vs } \\
\text { Rivaroxaban } \\
0.42(0.24-0.78) \\
p=0.370\end{array}$ & $\begin{array}{l}\text { Continuous D-dimer } \\
\text { prognostic factor for } \\
\text { composite outcome: } \\
\text { univariate OR } 1.15 \\
(1.03-1.29) p=0.015 \\
\text { multivariate OR } 1.13 \\
(1.0-1.28) p=0.048\end{array}$ \\
\hline $\begin{array}{l}\text { Mjelva, } 2016 \\
\text { [42] }\end{array}$ & $\begin{array}{l}\text { CAD } \\
(44.3 \% \\
\text { AMI) }\end{array}$ & 871 & 69.5 & 38.7 & P & 84 & $\begin{array}{l}\text { All-cause mortality; a } \\
\text { combined endpoint } \\
\text { consisting of death or } \\
\text { recurrent non-fatal Ml; } \\
\text { recurrent non-fatal Ml } \\
\text { alone. }\end{array}$ & $\begin{array}{l}194(106-437) \\
\mu \mathrm{g} / \mathrm{L} \\
\text { Median D- } \\
\text { dimer in survi- } \\
\text { vors vs non- } \\
\text { survivors } \\
\text { were for } 153 \text { vs } \\
346 \mu \mathrm{g} / \mathrm{L} \text { ( } p< \\
0.001 \text { ) }\end{array}$ & $\begin{array}{l}\text { D-dimer above } 436 \mu \mathrm{g} / \\
\mathrm{L} \text { independently } \\
\text { predicted mortality (4th } \\
\text { vs } 1 \text { st quartile HR } 1.83 \\
{[95 \% \mathrm{Cl} 1.20-2.78], p=} \\
0.005 \text { ) } \\
\text { Death or MI (4th vs 1st } \\
\text { quartile HR } 1.38 \text { [95\% Cl } \\
0.96-1.98], p=0.08) \\
\text { Recurrent } \mathrm{MI} \text { (4th vs 1st } \\
\text { quartile HR } 0.70 \text { [95\% Cl } \\
0.43-1.15], p=0.16 \text { ) }\end{array}$ \\
\hline $\begin{array}{l}\text { Gong } 2016 \\
{[43]}\end{array}$ & $\begin{array}{l}\text { CAD } \\
(29.2 \% \\
\text { AMI) }\end{array}$ & 2209 & 58.58 & 25.9 & P & 18 & $\begin{array}{l}\text { Cardiac death, nonfatal } \\
\text { Ml, recurrence of MI, } \\
\text { and stroke }\end{array}$ & $\begin{array}{l}\text { Tertiles }(\mu \mathrm{g} / \\
\mathrm{mL}) \\
1:<0.23, n= \\
816 ; \\
2: 0.23-0.36 \\
n=629 ; \\
3:>0.36, n= \\
764 .\end{array}$ & $\begin{array}{l}\text { D-dimer was linked to } \\
\text { the severity of CAD } \\
\text { (95\% Cl: } 1.20-6.84, p= \\
\text { 0.005) } \\
\text { Continuous D-dimer } \\
\text { predictor of total out- } \\
\text { come (HR }=1.22,95 \% \\
\text { Cl: } 1.09-1.37, p=0.001) \text {. }\end{array}$ \\
\hline $\begin{array}{l}\text { Charoensri } \\
2011 \text { [44] }\end{array}$ & $\begin{array}{l}\text { ACS } \\
(61 \% \\
\text { AMI) }\end{array}$ & 74 & 66 & 54.1 & R & $\begin{array}{l}\text { In- } \\
\text { hospital }\end{array}$ & $\begin{array}{l}\text { CHF, arrhythmias and } \\
\text { death }\end{array}$ & $\begin{array}{l}\text { D-dimer levels } \\
\text { ( } \mu \mathrm{g} / \mathrm{L}) \\
\text { CHF: } 1475 \text { vs } \\
\text { No CHF } 385 ; \\
\text { Arrhythmia }\end{array}$ & $\begin{array}{l}\text { D-dimer levels } \\
\text { correlated with } \\
\text { complication of ACS } \\
\text { (CHF; } p<0.001 \text {, } \\
\text { arrhythmia; } p=0.007\end{array}$ \\
\hline
\end{tabular}


Table 1 Clinical characteristics of studies investigating major adverse cardiac events and all-cause mortality included in the systematic review (Continued)

\begin{tabular}{|c|c|c|c|c|c|c|c|c|c|}
\hline Author/ year & Setting & $\mathrm{N}$ & Age (years) & $\begin{array}{l}\text { Women } \\
\text { (\%) }\end{array}$ & $\begin{array}{l}\text { Study } \\
\text { Design }\end{array}$ & $\begin{array}{l}\text { FU } \\
\text { (months) }\end{array}$ & Endpoints & D-dimer & Main findings \\
\hline & & & & & & & & $\begin{array}{l}5422 \text { vs No } \\
\text { arrhythmia 550; } \\
\text { Death } 5118 \text { vs } \\
\text { No death } 2550\end{array}$ & $\begin{array}{l}\text { and death; } p=0.009 \text { ). } \\
\text { D-dimer was signifi- } \\
\text { cantly increased with } \\
\text { the number of coronary } \\
\text { arteries affected ( } p= \\
\text { 0.03). }\end{array}$ \\
\hline $\begin{array}{l}\text { Brugger- } \\
\text { Andersen } \\
2008 \text { [45] }\end{array}$ & $\begin{array}{l}\text { STEMI } \\
(15 \%), \\
\text { NSTEMI } \\
(29,3 \%), \\
\text { UA } \\
(9,4 \%), \\
\text { No ACS } \\
(46,3 \%)\end{array}$ & 871 & 69.6 & 39 & P & 24 & $\begin{array}{l}\text { All-cause mortality, } \\
\text { CVEs (cardiac death or } \\
\text { recurrent positive } \\
\text { troponin T) }\end{array}$ & $\begin{array}{l}\text { Quartiles of D- } \\
\text { dimer }(\mu \mathrm{g} / \mathrm{l}) \text { : } \\
\text { Q1 }<106 \\
\text { Q2 } \geq 106-191 \\
\text { Q3 } \geq 191-438 \\
\text { Q4 } \geq 438\end{array}$ & $\begin{array}{l}\text { In the univariate } \\
\text { analysis highest } \mathrm{D} \text { - } \\
\text { dimer quartile predicted } \\
\text { all-cause mortality com- } \\
\text { pared with the lowest } \\
\text { quartile (Q1) (OR } 7.78 \\
\text { [95\%IC, 3.95-15.33], } \mathrm{p}< \\
0.001) \text {, but not con- } \\
\text { firmed at multivariable } \\
\text { logistic regression ana- } \\
\text { lysis (OR 1.80 [95\%|C, } \\
0.81 \text { to } 3.97] ; p=0.148) \text {. }\end{array}$ \\
\hline $\begin{array}{l}\text { Prisco } 2001 \\
{[46]}\end{array}$ & $\begin{array}{l}\text { CAD } \\
(52,9 \% \\
\text { AMI) }\end{array}$ & 54 & $\begin{array}{l}60(44-75) \\
65(38-81)\end{array}$ & 11.1 & $P$ & 18 & Restenosis & $\begin{array}{l}\text { D-dimer before } \\
\text { PCl: AMl } \\
\text { (group 1) } 55 \\
\text { ng/mL vs } \\
\text { elective PCl } \\
\text { (group 2) } 29.0 \\
\text { ng/mL, } p< \\
0.001\end{array}$ & $\begin{array}{l}\text { In group 1, D-dimer } \\
\text { levels at the end of the } \\
\text { procedure were higher } \\
\text { in patients with resten- } \\
\text { osis than in those with- } \\
\text { out }(p<0.005) \text {. } \\
\text { Increased D-dimer in } \\
\text { patients with restenosis } \\
(61 \%) \text { than those with- } \\
\text { out }(25 \%, p<0.05) \text {. }\end{array}$ \\
\hline $\begin{array}{l}\text { Oldgren } \\
2001 \text { [12] }\end{array}$ & ACS & 320 & 66 & - & P & 29 & $\begin{array}{l}\text { Death, MI, and } \\
\text { refractory angina } \\
\text { during and after } \\
\text { anticoagulant } \\
\text { treatment in unstable } \\
\text { CAD }\end{array}$ & $\begin{array}{l}<82 \mu \mathrm{g} / \mathrm{L}(\mathrm{N}= \\
105) ; 82- \\
149 \mu \mathrm{g} / \mathrm{L}(\mathrm{N}= \\
106) ; \\
>149 \mu \mathrm{g} / \mathrm{L} \\
(N=103)\end{array}$ & $\begin{array}{l}\text { No difference in clinical } \\
\text { outcome at } 72 \mathrm{~h}, 7 \text { days } \\
\text { and } 30 \text { days. During } \\
\text { long-term follow-up, } \\
\text { there was a relation be- } \\
\text { tween higher baseline } \\
\text { levels of D-dimer and } \\
\text { increased mortality ( } p= \\
0.003 \text { ). }\end{array}$ \\
\hline
\end{tabular}

Study design: $A$ ambispective, $P$ prospective, $R$ retrospective

Sincluding recurrent $\mathrm{MI}$, required renal replacement therapy, stent thrombosis, bleeding and length of hospital stay, hospital costs, and mortality and long-term CVEs (mortality, stent restenosis, non-fatal MI and TVR)

$A C S$ acute coronary syndrome, $A M I$ acute myocardial infarction, $A U C$ area under curve, $C A B G$ coronary artery bypass grafting, $C A D$ coronary artery disease, $C H F$ congestive heart failure, CI-AKI Contrast-induced acute kidney injury, CV cardiovascular, CVEs cardiovascular events, ESRD end-stage renal disease, HF heart failure, HFPEF heart failure with preserved ejection fraction, HFrEF heart failure with reduced ejection fraction, HR hazard ratio, NSTEMI non-ST segment elevation myocardial infarction, NT-proBNP N-terminal pro-B-type natriuretic peptide, OR odds ratio, PCI percutaneous coronary intervention, STEMI ST segment elevation myocardial infarction, TIA transient ischemic attack, TIMI Thrombolysis in Myocardial Infarction, TVR target vessel revascularization, UA unstable angina

heterogeneous population mixing STEMI, NSTEMI, unstable angina and no ACS patients.

\section{Results of meta-analysis}

Five studies satisfied inclusion criteria and were included in the metanalysis: 4 prospective and 1 retrospective studies (Table 1) with a total of 8616 patients. Only studies reporting HR for continuous values were formally analysed. Median follow-up was 13.2 months with a total of 626 CVEs. The weighted mean age was 61.9 years. The percentage of women ranged from 25.9 to $100 \%$ (in one study). The pooled HR for D-dimer levels and CVEs was 1.264 (95\% CI 1.134-1.409) (Fig. 1).

\section{No-reflow phenomenon in STEMI patients}

We also performed a systematic search using "D-dimer" and "no-reflow". After screening, 7 potentially eligible studies were identified (3 prospective, 3 retrospective and 1 ambispective) (Supplementary Fig. 2). A total of 4195 STEMI patients were included, with mean age ranged from 52.6 to 64.0 mean years (female patients from 16 to $53.7 \%)$. Overall, 1327 patients (31.6\%) had no-reflow phenomenon (Table 2). Five out of 7 studies showed a positive correlation of D-dimer levels with noreflow phenomenon. Given the high variability in the methods of each study (cut-off of D-dimer used, measures of association) a formal analysis was not performed. 


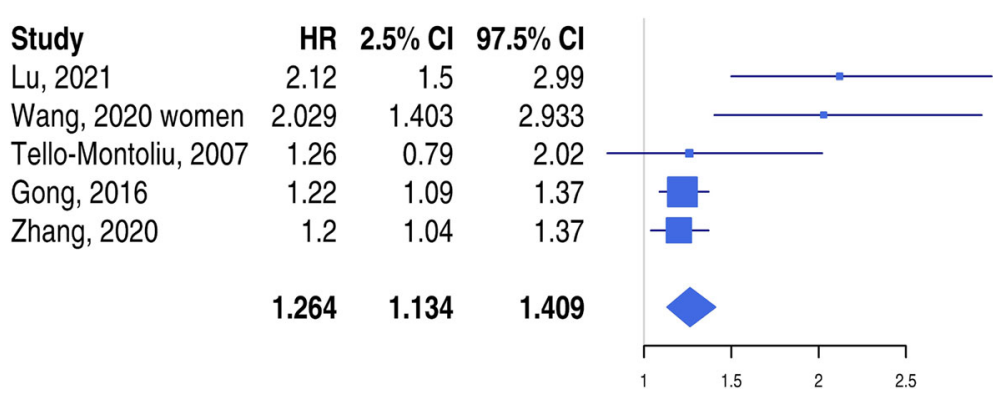

Fig. 1 Forest plot of the hazard ratio for the risk of composite endpoints according to D-dimer values in patients with acute MI. HR: hazard ratio; Cl: confidence interval

\section{Discussion}

Our study shows that most studies including patients with ischemic heart disease showed a consistent association between D-dimer levels and worse clinical outcomes. This association was particularly evident in studies investigating in-hospital adverse events.

The rationale of investigating D-dimer levels in CAD patients relies on the persistent hypercoagulable state described in MI patients, which may lead to worse clinical outcomes in these patients through several mechanisms [14]. Clotting activation may contribute to thrombus growth and no-reflow phenomenon, along with impaired thrombolysis which has been frequently described in CAD patients [49], all factors contributing to an increased CAD severity and myocardial injury (Fig. 2).

Reperfusion fails to occur after thrombolytic therapy in approximate $40 \%$ of patients, as shown by a study including 427 STEMI patients undergo rescue PCI [50]. Indeed, high D-dimer levels have been shown to be significantly higher in lysis-resistant thrombi from STEMI patients [51].

Table 2 Clinical characteristics of studies investigating no-reflow phenomenon included in the systematic review

\begin{tabular}{|c|c|c|c|c|c|c|c|c|c|c|c|}
\hline \multirow{2}{*}{$\begin{array}{l}\text { Author/ } \\
\text { year }\end{array}$} & \multirow[t]{2}{*}{$\mathrm{N}^{\mathrm{a}}$} & \multirow{2}{*}{$\begin{array}{l}\begin{array}{l}\text { Age } \\
\text { (years) }\end{array} \\
\end{array}$} & \multirow{2}{*}{$\begin{array}{l}\text { Women } \\
(\%)\end{array}$} & \multirow{2}{*}{$\begin{array}{l}\text { Study } \\
\text { design }\end{array}$} & \multirow[t]{2}{*}{ Events } & \multicolumn{2}{|c|}{ D-dimer levels } & \multirow{2}{*}{$\begin{array}{l}\text { Odds } \\
\text { Ratio }\end{array}$} & \multirow{2}{*}{$\begin{array}{l}\text { Low } \\
\mathrm{Cl}\end{array}$} & \multirow{2}{*}{$\begin{array}{l}\text { High } \\
\mathrm{Cl}\end{array}$} & \multirow[t]{2}{*}{ Main findings } \\
\hline & & & & & & $\begin{array}{l}\text { No-reflow } \\
\text { group }\end{array}$ & $\begin{array}{l}\text { Control } \\
\text { group }\end{array}$ & & & & \\
\hline $\begin{array}{l}\text { Gong } \\
2020 \\
{[47]}\end{array}$ & 229 & 63.7 & 17 & $\mathrm{R}$ & 28 & $\begin{array}{l}1600 \pm \\
1400 \mathrm{ng} / \\
\mathrm{mL}\end{array}$ & $\begin{array}{l}500 \pm \\
600 \mathrm{ng} / \\
\mathrm{mL}\end{array}$ & 2.520 & 1.160 & 5.470 & $\begin{array}{l}\text { D-dimer level can independently predict no- } \\
\text { reflow after PCI. D-dimer value of } 530 \mathrm{ng} / \mathrm{mL} \\
\text { was an effective cut-off point for postproce- } \\
\text { dural no-reflow with } 85.7 \% \text { of sensitivity and } \\
67.7 \% \text { of specificity ( } A \cup C=0.78 ; p=0.049 \text { ). }\end{array}$ \\
\hline $\begin{array}{l}\text { Huang } \\
2020 \\
{[18]}\end{array}$ & 1165 & 63.5 & 17 & $\mathrm{R}$ & 165 & $\begin{array}{l}\geq 800 \mathrm{ng} / \\
\mathrm{mL}\end{array}$ & $\begin{array}{l}<800 \\
\mathrm{ng} / \mathrm{mL}\end{array}$ & 1.399 & 0.929 & 2.106 & $\begin{array}{l}\text { D-dimer group had more frequently no-reflow } \\
(13.1 \% \text { vs. } 18.8 \% . p=0.028) \text {. }\end{array}$ \\
\hline $\begin{array}{l}\text { Cheng } \\
2019 \\
{[48]}\end{array}$ & 218 & 58.7 & 17.5 & $\mathrm{R}$ & 39 & $\begin{array}{l}410.3 \pm \\
237.2 \mathrm{ng} / \\
\mathrm{mL}\end{array}$ & $\begin{array}{l}536.9 \pm \\
291.7 \\
\mathrm{ng} / \mathrm{mL}\end{array}$ & 1.001 & 1.000 & 1.003 & $\begin{array}{l}\text { No-reflow patients were older, diabetics, with } \\
\text { longer pain-to balloon time, lower blood pres- } \\
\text { sure, higher platelet count and higher levels of } \\
\text { D-dimer and Cystatin C. }\end{array}$ \\
\hline $\begin{array}{l}\text { Zhang } \\
2018 \\
{[22]}\end{array}$ & 926 & 52.6 & 53.7 & $P$ & 435 & $\begin{array}{l}508.5 \pm \\
254.7 \mathrm{ng} / \\
\mathrm{mL}\end{array}$ & $\begin{array}{l}272.0 \pm \\
218.9 \\
\mathrm{ng} / \mathrm{mL}\end{array}$ & 2.563 & 1.910 & 3.439 & $\begin{array}{l}\text { Multivariate OR for predicting no-reflow for D- } \\
\text { dimer above mean }(383.1 \mathrm{ng} / \mathrm{mL}) \text {. }\end{array}$ \\
\hline $\begin{array}{l}\text { Gao } \\
2018 \\
{[23]}\end{array}$ & 822 & 62.5 & 46.1 & $P$ & 418 & $\begin{array}{l}533.0 \pm \\
244.0 \mathrm{ng} / \\
\mathrm{mL}\end{array}$ & $\begin{array}{l}323.4 \pm \\
224.4 \\
\mathrm{ng} / \mathrm{mL}\end{array}$ & 4.212 & 2.973 & 5.967 & $\begin{array}{l}\text { Diabetic patients with high D-dimer levels } \\
\text { showed higher risk of no-reflow. Sensitivity of } \\
\text { high plasma D-dimer levels in predicting no- } \\
\text { reflow was } 0.766 \text {. }\end{array}$ \\
\hline $\begin{array}{l}\text { Sarli } \\
2015 \\
{[25]}\end{array}$ & 266 & 64 & 38 & $\mathrm{P}$ & 63 & $\begin{array}{l}686 \pm \\
236 \mu \mathrm{g} / \mathrm{l}\end{array}$ & $\begin{array}{l}418 \pm \\
164 \mu \mathrm{g} / \mathrm{l}\end{array}$ & 1.005 & 1.003 & 1.007 & $\begin{array}{l}\text { D-dimer levels predicted no-reflow (OR: 1.005; } \\
95 \% \mathrm{Cl}: 1.003-1.007 ; p<0.001 \text { ). Optimal cut-off } \\
\text { for no-reflow was } 549 \mu \mathrm{g} / \mathrm{l} \text {. }\end{array}$ \\
\hline $\begin{array}{l}\text { Erkol } \\
2014 \\
{[15]}\end{array}$ & 569 & 56 & 16 & A & 179 & $\begin{array}{l}720(280- \\
1490) \mathrm{mg} / \\
L\end{array}$ & $\begin{array}{l}350 \\
(170- \\
620) \\
\mathrm{mg} / \mathrm{L}\end{array}$ & 1.640 & 1.260 & 2.140 & $\begin{array}{l}\text { D-dimer (per each } 1 \mathrm{mg} / \mathrm{L} \text { increase) predictor } \\
\text { of angiographic no-reflow }(p<0.001) \text {. }\end{array}$ \\
\hline
\end{tabular}




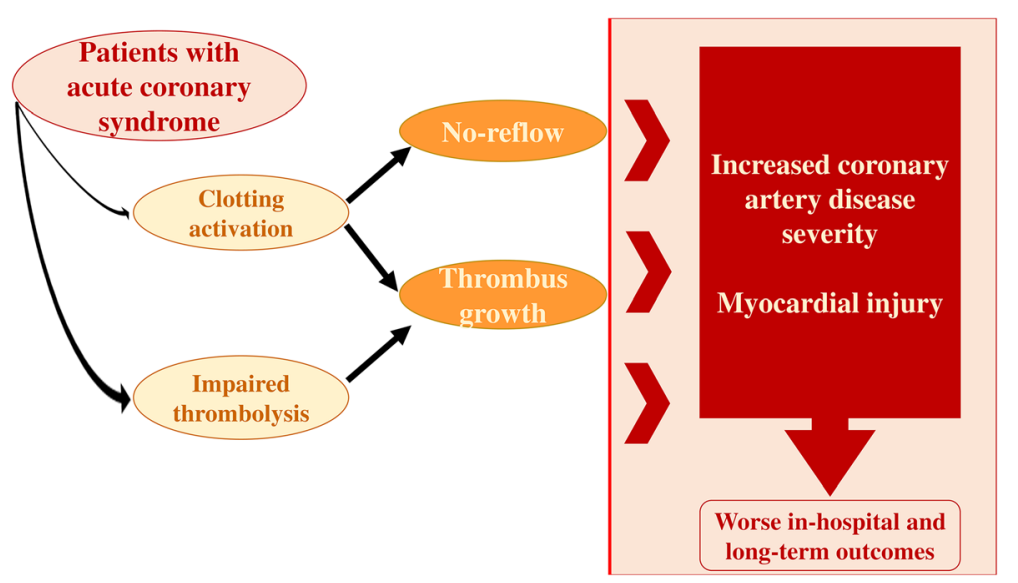

Fig. 2 Pathophysiological mechanisms linking clotting activation and impairment of thrombolysis with the severity of acute coronary syndrome presentation and clinical outcomes

The pathogenesis of no-reflow remains unclear, but it is likely to be multifactorial, including endothelial damage platelet and clotting activation leading to thrombus formation at the level of small vessels [52]. Few studies investigated the occurrence of no-reflow in STEMI patients undergoing $\mathrm{PCI}$ and its relationship with $\mathrm{D}$-dimer $[15,22,23,25,47,48]$. In 229 consecutive STEMI patients, X. Gong et al. [47] found that a D-dimer value of $530 \mathrm{ng} / \mathrm{mL}$ (FEU) was associated with postprocedural no-reflow, with $85.7 \%$ of sensitivity and $67.7 \%$ of specificity (area under the curve $[\mathrm{AUC}]=0.78 ; p=0.049$ ). Similarly, Sarli et al. found that optimal D-dimer cut-off value for predicting no-reflow was $549 \mu \mathrm{g} / \mathrm{l}$ (FEU) [25]. In 218 STEMI patients, Cheng et al. [48] found that noreflow patients were older, diabetics, with longer pain-to balloon time, lower blood pressure, higher platelet counts and higher levels of D-dimer and Cystatin C than patients without no-reflow.

\section{Risk stratification using D-dimer}

In the past decades, an increasing number of biomarkers have been tested to improve cardiovascular risk stratification in patients with ACS $[6,53]$. In particular, some studies suggested that the use of a combination of multiple biomarkers may improve risk stratification. This is the case of D-dimer, which if used alone is currently recommended to only exclude acute venous thromboembolism, but its addition to other biomarkers/risk scores gave promising results.

Indeed, the addition of D-dimer has been investigated to some biomarkers such as C-reactive protein (CRP), NT-proBNP and clinical scores, such as the Global Registry of Acute Coronary Events (GRACE) risk score has been investigated. Fu et al. included D-dimer levels $\geq 2.4 \mathrm{mg} / \mathrm{L}$ FEU in a risk score model together with CRP, left ventricular ejection fraction, age $\geq 65$ years old and heart rate [32]. In ROC curve analysis, this model demonstrated a good power in predicting in-hospital mortality $(\mathrm{AUC}=0.895,95 \%$ CI $0.814-0.96 ; p<0.001)$, better than the predictive power of the GRACE risk score alone $(\mathrm{AUC}=0.754,95 \%$ CI 0.641-0.868; $\mathrm{p}<0.001)$. D-dimer has been tested combined to GRACE score also in the study by Lin et al. who found that the combination of NT-pro-BNP and D-dimer improved the predictive accuracy of GRACE score for all-cause death [29]. In 5923 ACS patients undergoing PCI, GRACE score combined with $\mathrm{D}$-dimer achieved a better prognostic performance than GRACE score, and D-dimer could significantly improve the prognostic performance of GRACE score [35]. This result is in keeping with that described by $\mathrm{Yu}$ et al., in which [35] D-dimer levels significantly improved the prognostic performance of GRACE score.

\section{Management and therapeutic implications}

Altogether, current evidence suggest that D-dimer may identify patients at higher risk for a more severe CAD and worse prognosis. Of note, clotting activation is not lowered by antiplatelet therapy [54], as shown by previous studies reporting no effect of aspirin and DAPT in reducing D-dimer levels [55]. Conversely, oral and parenteral anticoagulation, in addition or not to DAPT, has been demonstrated to decrease D-dimer levels in patients with MI $[41,56,57]$. Thus, ATLAS ACS 2-TIMI 51 and COMPASS trials have recently proved the utility of adding low-dose anticoagulation to antiplatelet medications. In ATLAS ACS 2-TIMI 51, introduction of lowdose rivaroxaban therapy $(2.5 \mathrm{mg}$ or $5 \mathrm{mg}$ twice daily, with $93 \%$ of patients on DAPT) has shown to be effective in reducing the risk of death from cardiovascular causes, myocardial infarction, or stroke [58], even if the use of rivaroxaban increased the risk of major bleeding and intracranial haemorrhages. Similarly, the COMPASS 
trial demonstrated that rivaroxaban plus aspirin was effective in decreasing the primary outcome of cardiovascular death, stroke or MI with a $22 \%$ reduction in the net clinical benefit endpoint (defined as the composite of primary outcome, fatal bleeding and symptomatic bleeding into a critical organ/area) [59]. Moreover, a subanalysis of the COMPASS trial has shown that patients with high thrombotic profile (i.e., peripheral arterial disease) may benefit more from adding anticoagulation therapy to reduce residual thrombotic risk.

However, these studies did not stratified patients according to D-dimer levels. Data from the literature let to hypothesize that the benefit conferred by an association therapy of oral anticoagulation and antiplatelet therapy may be even more evident in patients with the features of clotting activation (i.e. high D-dimer). This aspect would deserve a specific investigation.

Another group of patients who deserve particular attention is represented by patients presenting with noreflow. Preliminary data from the literature suggest an association between high-D-dimer levels and no-reflow. Moreover, in MI patients who cannot undergo cardiac catheterization, D-dimer concentrations may also be helpful to predict severity of coronary disease [60].

Finally, D-dimer levels might be helpful in identifying MI patients at high risk of complications and worse outcome. In Fig. 3, we propose a D-dimer guided strategy to tailored therapy in patients at higher thrombotic risk such as those with MI undergoing PCI. Firstly, MI patients with high D-dimer levels may benefit from specific pharmacological management and technical procedures to reduce the probability of no-reflow phenomenon. These include the use of glycoprotein IIb/IIIa inhibitors, higher heparin dose and direct stenting without predilation [61-64]. Those high-risk patients might also take advantage from prolonged parenteral anticoagulation after PCI during in-hospital staying to reduce early complications. At discharge, patients presenting with highrisk features, including presence of comorbidities, multivessel disease, age $>65$ years, recurrent MI, multiple stent insertion, may benefit from adding a low dose of oral anticoagulant agent to conventional antiplatelet therapy to reduce the residual thrombotic risk (i.e., rivaroxaban $2.5 \mathrm{mg}$ twice daily).

Study limitations. A formal analysis for D-dimer against no-reflow phenomenon was not performed given the high variability in the methods of each study (cut-off of D-dimer used, measures of association) was not performed.

\section{Conclusions}

In conclusion, given its association with CVEs, the use of D-dimer may help physicians to improve outcome and manage residual thrombotic risk in MI

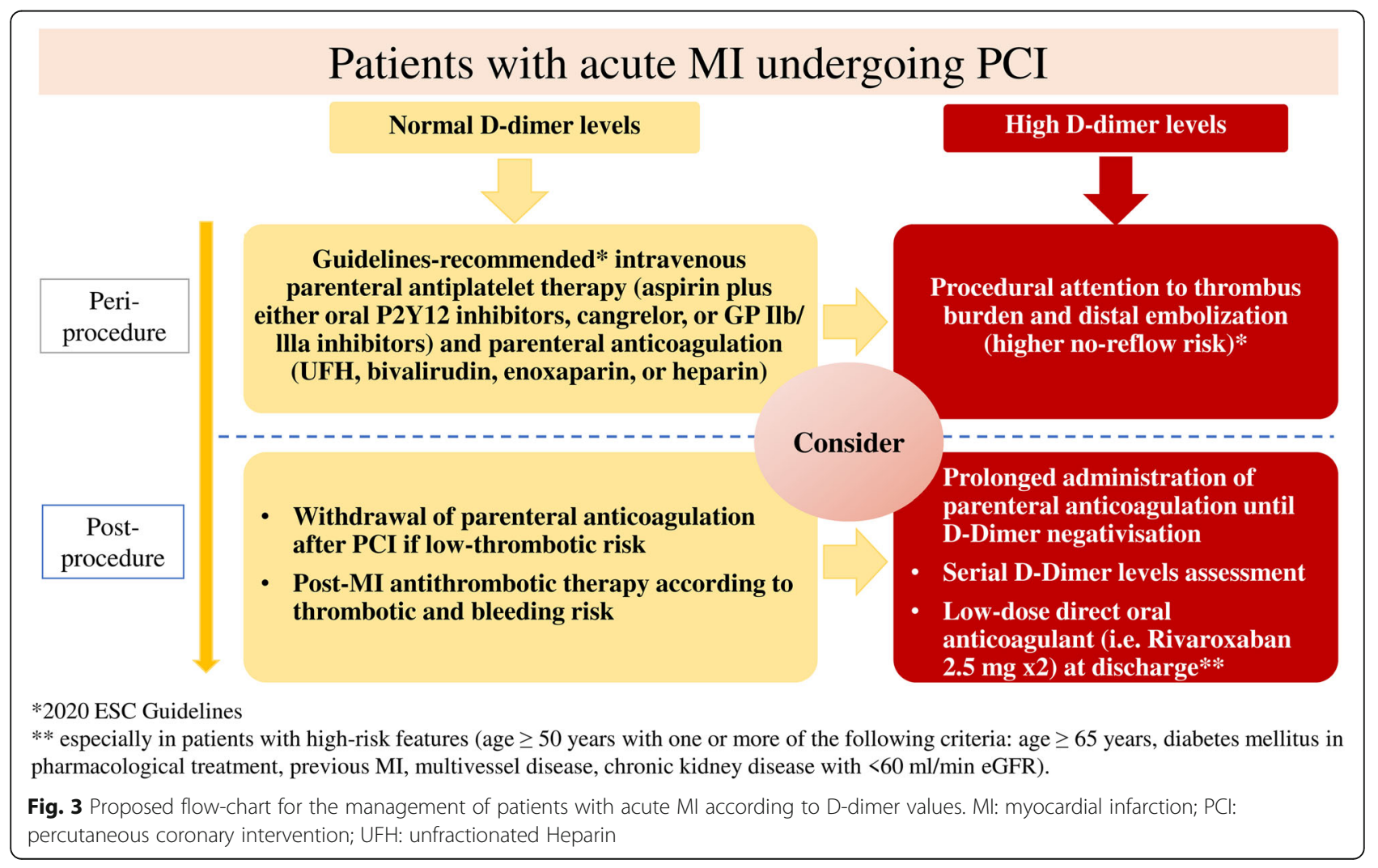


patients. D-dimer may therefore represent a valuable guide in the difficult decision-making process on the use of oral anticoagulation in addition to currently recommended antithrombotic therapy in patients with ACS.

\section{Abbreviations}

CAD: Coronary artery disease; Ml: Myocardial infarction; PCl: Percutaneous coronary intervention; CVEs: Cardiovascular events; STEMI: ST segment elevation myocardial; NSTEMI: Non-ST segment elevation myocardial infarction; ACS: Acute coronary syndrome; CRP: C-reactive protein; GRACE: Global registry of acute coronary events; TIMI: Thrombolysis in myocardial infarction

\section{Supplementary Information}

The online version contains supplementary material available at https://doi. org/10.1186/s12959-021-00354-y.

Additional file 1. Supplementary data.

\section{Acknowledgements}

this research did not receive any specific grant from funding agencies in the public, commercial, or not-for-profit sectors.

\section{Authors' contributions}

F.G.B. and D.P. are the guarantors of the manuscript and responsible for project administration, study concept, design and literature search. Data collection/interpretation/analysis: F.G.B, D.P., A.F. and G.T. Manuscript writing/ revisions, validation, visualization and drafting of figures: F.G.B., D.P., A.F., C.G., F.V., P.P. and G.T. The authors read and approved the final manuscript.

\section{Funding}

None.

\section{Availability of data and materials}

The datasets used and/or analysed during the current study are available from the corresponding author on reasonable request.

\section{Declarations}

\section{Ethics approval and consent to participate}

Not applicable.

\section{Consent for publication}

Not applicable.

\section{Competing interests}

The authors declare that they have no competing interests.

\section{Author details}

${ }^{1}$ Department of Clinical Internal, Anesthesiological, and Cardiovascular Sciences, Sapienza University of Rome, Viale del Policlinico 155, 00161 Rome, Italy. 'Department of General and Specialized Surgery "Paride Stefanini", Sapienza University of Rome, Viale del Policlinico 155, 00161 Rome, Italy. ${ }^{3}$ Department of Economics and Finance, University of Rome "Tor Vergata", Via Columbia 2, 00133 Rome, Italy.

Received: 23 October 2021 Accepted: 29 November 2021 Published online: 18 December 2021

\section{References}

1. Roth GA, Huffman MD, Moran AE, Feigin V, Mensah GA, Naghavi M, et al. Global and regional patterns in cardiovascular mortality from 1990 to 2013. Circulation. 2015;132(17):1667-78. https://doi.org/10.1161/CIRCULATIONAHA.114.008720.

2. Jernberg $T$, Hasvold $P$, Henriksson M, Hjelm $H$, Thuresson M, Janzon M. Cardiovascular risk in post-myocardial infarction patients: nationwide real world data demonstrate the importance of a long-term perspective. Eur Heart J. 2015;36(19):1163-70. https://doi.org/10.1093/eurheartj/ehu505.
3. Townsend N, Wilson L, Bhatnagar P, Wickramasinghe K, Rayner M, Nichols M. Cardiovascular disease in Europe: epidemiological update 2016. Eur Heart J. 2016;37(42):3232-45. https://doi.org/10.1093/eurheartj/ehw334.

4. Global, regional, and national age-sex-specific mortality for 282 causes of death in 195 countries and territories, 1980-2017: a systematic analysis for the Global Burden of Disease Study 2017. Lancet. 2018;392(10159):1736-88. https://doi.org/10.1016/S0140-6736(18)32203-7.

5. Dhingra R, Vasan RS. Biomarkers in cardiovascular disease: statistical assessment and section on key novel heart failure biomarkers. Trends Cardiovasc Med. 2017;27(2):123-33. https://doi.org/10.1016/j.tcm.2016. 07.005

6. Collet JP, Thiele H, Barbato E, Barthélémy O, Bauersachs J, Bhatt DL, et al. ESC guidelines for the management of acute coronary syndromes in patients presenting without persistent ST-segment elevation. Eur Heart J. 2020;2020(3):4418. https://doi.org/10.15829/1560-4071-2021-4418.

7. Amsterdam EA, Wenger NK, Brindis RG, Casey DE Jr, Ganiats TG, Holmes DR Ir, et al. AHA/ACC guideline for the Management of Patients with non-STelevation acute coronary syndromes: a report of the American College of Cardiology/American Heart Association task force on practice guidelines. Am Coll Cardiol. 2014;64:e139-228. https://doi.org/10.1016/j.jacc.2014.09.017.

8. Galvani M, Ferrini D, Ottani F, Eisenberg PR. Early risk stratification of unstable angina/non-Q myocardial infarction: biochemical markers of coronary thrombosis. Int J Cardiol. 1999;68(Suppl 1):S55-61. https://doi.org/1 0.1016/S0167-5273(98)00292-7.

9. Ardissino D, Merlini PA, Gamba G, Barberis P, Demicheli G, Testa S, et al. Thrombin activity and early outcome in unstable angina pectoris. Circulation. 1996;93(9):1634-9. https://doi.org/10.1161/01.CIR.93.9.1634.

10. Merlini PA, Bauer KA, Oltrona L, Ardissino D, Cattaneo M, Belli C, et al. Persistent activation of coagulation mechanism in unstable angina and myocardial infarction. Circulation. 1994;90(1):61-8. https://doi.org/10.1161/ 01.CIR.90.1.61

11. Giannitsis E, Mair J, Christersson C, Siegbahn A, Huber K, Jaffe AS, et al. How to use D-dimer in acute cardiovascular care. Eur Heart J Acute Cardiovasc Care. 2017:6(1):69-80. https://doi.org/10.1177/2048872615610870.

12. Oldgren J, Linder R, Grip L, Siegbahn A, Wallentin L. Coagulation activity and clinical outcome in unstable coronary artery disease. Arterioscler Thromb Vasc Biol. 2001;21(6):1059-64. https://doi.org/10.11 61/01.ATV.21.6.1059.

13. Soomro AY, Guerchicoff A, Nichols DJ, Suleman J, Dangas GD. The current role and future prospects of D-dimer biomarker. Eur Heart J Cardiovasc Pharmacother. 2016;2(3):175-84. https://doi.org/10.1093/ehjcvp/pvv039.

14. Christersson C, Oldgren J, Bylock A, Siegbahn A, Wallentin L. Early decrease in coagulation activity after myocardial infarction is associated with lower risk of new ischaemic events: observations from the ESTEEM trial. Eur Heart J. 2007:28(6):692-8. https://doi.org/10.1093/eurheartj/ehl564.

15. Erkol A, Oduncu V, Turan B, Kılıçgedik A, Sırma D, Gözübüyük G, et al. The value of plasma D-dimer level on admission in predicting no-reflow after primary percutaneous coronary intervention and long-term prognosis in patients with acute ST segment elevation myocardial infarction. J Thromb Thrombolysis. 2014;38(3):339-47. https://doi.org/10.1007/s11239-013-1044-3.

16. Rezkalla SH, Dharmashankar KC, Abdalrahman IB, Kloner RA. No-reflow phenomenon following percutaneous coronary intervention for acute myocardial infarction: incidence, outcome, and effect of pharmacologic therapy. J Interv Cardiol. 2010;23(5):429-36. https://doi.org/10.1111/j.1540-81 83.2010.00561x

17. Biccirè FG, Pastori $D$, Tanzilli A, Pignatelli P, Viceconte $N$, Barillà $F$, et al. Low serum albumin levels and in-hospital outcomes in patients with ST segment elevation myocardial infarction. Nutr Metab Cardiovasc Dis. 2021;31(10): 2904-11.

18. Huang D, Gao W, Wu R, Zhong X, Qian J, Ge J. D-dimer level predicts inhospital adverse outcomes after primary PCI for ST-segment elevation myocardial infarction. Int J Cardiol. 2020;305:1-4. https://doi.org/10.1016/j. ijcard.2020.02.010

19. Luo E, Wang D, Liu B, Hou J, Yan G, Tang C. The value of d-dimer level in predicting contrast-induced acute kidney injury in patients with acute STsegment elevation myocardial infarction after PCl. Clin Appl Thromb Hemost 2020;26:1076029620944492. https://doi.org/10.1177/1076029620944492.

20. Zhou Q, Xue Y, Shen J, Zhou W, Wen Y, Luo S. Predictive values of D-dimer for the long-term prognosis of acute ST-segment elevation infarction: a retrospective study in southwestern China. Medicine (Baltimore). 2020; 99(16):e19724. https://doi.org/10.1097/MD.0000000000019724. 
21. Lin KY, Chen HC, Jiang H, Wang SY, Chen HM, Wu ZY, et al. Predictive value of admission D-dimer for contrast-induced acute kidney injury and poor outcomes after primary percutaneous coronary intervention. BMC Nephrol. 2020;21(1):90. https://doi.org/10.1186/s12882-020-01743-7.

22. Zhang H, Qiu B, Zhang Y, Cao Y, Zhang X, Wu Z, et al. The value of preinfarction angina and plasma D-dimer in predicting no-reflow after primary percutaneous coronary intervention in ST-segment elevation acute myocardial infarction patients. Med Sci Monit. 2018;24:4528-35. https://doi. org/10.12659/MSM.909360

23. Gao R, Wang J, Zhang S, Yang G, Gao Z, Chen X. The value of combining plasma D-dimer and Endothelin-1 levels to predict no-reflow after percutaneous coronary intervention of ST-segment elevation in acute myocardial infarction patients with a type 2 diabetes mellitus history. Med Sci Monit. 2018;24:3549-56. https://doi.org/10.12659/MSM.908980.

24. Hansen $\mathrm{CH}$, Ritschel V, Andersen G, Halvorsen S, Eritsland J, Arnesen $\mathrm{H}$, et al. Markers of thrombin generation are associated with long-term clinical outcome in patients with ST-segment elevation myocardial infarction. Clin Appl Thromb Hemost. 2018;24(7):1088-94. https://doi.org/10.1177/107602 9618764847.

25. Sarli B, Akpek M, Baktir AO, Sahin O, Saglam H, Arinc $H$, et al. Impact of Ddimer level on postinterventional coronary flow and in-hospital MACE in STsegment elevation myocardial infarction. Herz. 2015;40(3):507-13. https:// doi.org/10.1007/s00059-013-4029-2.

26. Kikkert WJ, Claessen BE, Stone GW, Mehran R, Witzenbichler B, Brodie BR, et al. D-dimer levels predict ischemic and hemorrhagic outcomes after acute myocardial infarction: a HORIZONS-AMI biomarker substudy. J Thromb Thrombolysis. 2014;37(2):155-64. https://doi.org/10.1007/s11239013-0953-5.

27. Akgul O, Uyarel H, Pusuroglu H, Gul M, Isiksacan N, Turen S, et al. Predictive value of elevated D-dimer in patients undergoing primary angioplasty for ST elevation myocardial infarction. Blood Coagul Fibrinolysis. 2013;24(7):70410. https://doi.org/10.1097/MBC.0b013e3283610396.

28. Pineda J, Marín F, Marco P, Roldán V, Valencia J, Ruiz-Nodar JM, et al. The prognostic value of biomarkers after a premature myocardial infarction. Int J Cardiol. 2010;143(3):249-54. https://doi.org/10.1016/j.ijcard.2009.02.019.

29. Lu PJ, Gong XW, Liu Y, Tian FS, Zhang WJ, Liu YW, et al. Optimization of GRACE risk stratification by $\mathrm{N}$-terminal pro-B-type natriuretic peptide combined with D-dimer in patients with non-ST-elevation myocardial infarction. Am J Cardiol. 2021;140:13-9. https://doi.org/10.1016/j.amjcard.202 0.10 .050 .

30. Satilmisoglu MH, Ozyilmaz SO, Gul M, Ak Yildirim H, Kayapinar O, Gokturk K, et al. Predictive values of D-dimer assay, GRACE scores and TIMI scores for adverse outcome in patients with non-ST-segment elevation myocardial infarction. Ther Clin Risk Manag. 2017;13:393-400. https://doi.org/10.2147/ TCRM.S124794.

31. Tello-Montoliu A, Marín F, Roldán V, Mainar L, López MT, Sogorb F, et al. A multimarker risk stratification approach to non-ST elevation acute coronary syndrome: implications of troponin T, CRP, NT pro-BNP and fibrin D-dimer levels. J Intern Med. 2007;262(6):651-8. https://doi.org/10.1111/j.1365-2796.2 007.01871.X

32. Fu $Y$, Sun $H$, Guo Z, Xu L, Yang $X$, Wang $L$, et al. A risk score model to predict in-hospital mortality of patients with end-stage renal disease and acute myocardial infarction. Intern Emerg Med. 2020;16(4):905-12. https:// doi.org/10.1007/s11739-020-02529-3.

33. Wang P, Yao J, Xie Y, Luo M. Gender-specific predictive markers of poor prognosis for patients with acute myocardial infarction during a 6-month follow-up. J Cardiovasc Transl Res. 2020;13(1):27-38. https://doi.org/10.1007/ s12265-019-09946-6.

34. Zhang X, Wang S, Sun L, Fang S, Yu B. Prognostic value of D-dimer in acute myocardial infarction complicated by heart failure with preserved ejection fraction. ESC Heart Fail. 2020;7(6):4118-25. https://doi.org/10.1 002/ehf2.13027.

35. Yu T, Jiao Y, Song J, He D, Wu J, Sun Z, et al. Hospital mortality in acute coronary syndrome: adjustment of GRACE score by D-dimer enables a more accurate prediction in a prospective cohort study. BMC Cardiovasc Disord. 2019;19(1):252. https://doi.org/10.1186/s12872-019-1239-4.

36. Christersson C, Lindahl B, Berglund L, Siegbahn A, Oldgren J. The utility of coagulation activity for prediction of risk of mortality and cardiovascular events in guideline-treated myocardial infarction patients. Ups J Med Sci. 2017;122(4):224-33. https://doi.org/10.1080/03009734.201 7.1407849 .
37. Smid M, Dielis AW, Winkens M, Spronk HM, van Oerle R, Hamulyák K, et al Thrombin generation in patients with a first acute myocardial infarction. J Thromb Haemost. 2011;9(3):450-6. https://doi.org/10.1111/j.1538-7836.2010. 04162.x.

38. Kalaria VG, Zareba W, Moss AJ, Pancio G, Marder VJ, Morrissey JH, et al. Gender-related differences in thrombogenic factors predicting recurrent cardiac events in patients after acute myocardial infarction. The THROMBO Investigators. Am J Cardiol. 2000;85(12):1401-8. https://doi.org/10.1016/ S0002-9149(00)00785-2.

39. Chen $H$, Lu N, Zheng M. A high circulating FGF21 level as a prognostic marker in patients with acute myocardial infarction. Am J Transl Res. 2018; 10(9):2958-66.

40. Kosaki R, Minoura Y, Ogura K, Oishi Y, Tanaka L, Arai K, et al. Thrombomodulin can predict the incidence of second events in patients with acute coronary syndrome: single-center, retrospective cohort study. J Cardiol. 2018;72(6):494-500. https://doi.org/10.1016/j.jjcc.2018.05.006.

41. AlKhalfan F, Kerneis M, Nafee T, Yee MK, Chi G, Plotnikov A, et al. D-dimer levels and effect of rivaroxaban on those levels and outcomes in patients with acute coronary syndrome (an ATLAS ACS-TIMI 46 trial substudy). Am J Cardiol. 2018;122(9):1459-64. https://doi.org/10.1016/j.amjcard.2018.07.032.

42. Mjelva $\varnothing R$, Pönitz V, Brügger-Andersen T, Grundt $H$, Staines $H$, Nilsen DW. Long-term prognostic utility of pentraxin 3 and D-dimer as compared to high-sensitivity C-reactive protein and B-type natriuretic peptide in suspected acute coronary syndrome. Eur J Prev Cardiol. 2016;23(11):113040. https://doi.org/10.1177/2047487315619733.

43. Gong P, Yang SH, Li S, Luo SH, Zeng RX, Zhang Y, et al. Plasma d-dimer as a useful marker predicts severity of atherosclerotic lesion and short-term outcome in patients with coronary artery disease. Clin Appl Thromb Hemost. 2016;22(7):633-40. https://doi.org/10.1177/1076029616634885.

44. Charoensri N, Pornratanarangsi S. D-dimer plasma levels in NSTE-ACS patient. J Med Assoc Thail. 2011;94(Suppl 1):S39-45.

45. Brügger-Andersen T, Pönitz $V$, Staines $H$, Grundt $H$, Hetland $\varnothing$, Nilsen DW. The prognostic utility of D-dimer and fibrin monomer at long-term followup after hospitalization with coronary chest pain. Blood Coagul Fibrinolysis. 2008;19(7):701-7. https://doi.org/10.1097/MBC.0b013e32830b1512.

46. Prisco D, Antonucci E, Fedi S, Margheri M, Giglioli C, Comeglio M, et al. D-dimer increase after percutaneous transluminal angioplasty and clinical recurrence after primary revascularization in acute myocardial infarction? A pilot study. Clin Exp Med. 2001;1(4):219-24. https://doi.org/10.1007/s102380100006.

47. Gong X, Lei X, Huang Z, Song Y, Wang Q, Qian J, et al. D-dimer level predicts angiographic no-reflow phenomenon after percutaneous coronary intervention within 2-7 days of symptom onset in patients with ST-segment elevation myocardial infarction. J Cardiovasc Transl Res. 2020;14(4):728-34. https://doi.org/10.1007/s12265-020-09991-6.

48. Cheng C, Liu X-B, Bi S-J, Lu Q-H, Zhang J. Serum cystatin C levels relate to no-reflow phenomenon in percutaneous coronary interventions in STsegment elevation myocardial infarction. PLoS One. 2019;14(8):e0220654. https://doi.org/10.1371/journal.pone.0220654.

49. Okafor ON, Gorog DA. Endogenous fibrinolysis: an important mediator of Thrombus formation and cardiovascular risk. J Am Coll Cardiol. 2015;65(16): 1683-99. https://doi.org/10.1016/j.jacc.2015.02.040.

50. Gershlick AH, Stephens-Lloyd A, Hughes S, Abrams KR, Stevens SE, Uren NG, et al. Rescue angioplasty after failed thrombolytic therapy for acute myocardial infarction. N Engl J Med. 2005;353(26):2758-68. https://doi.org/1 0.1056/NEJMoa050849.

51. Sambola A, García Del Blanco B, Ruiz-Meana M, Francisco J, Barrabés JA, Figueras J, et al. Increased von Willebrand factor, P-selectin and fibrin content in occlusive thrombus resistant to lytic therapy. Thromb Haemost. 2016;115(6):1129-37. https://doi.org/10.1160/TH15-12-0985.

52. Kloner RA, Ganote CE, Jennings RB. The "no-reflow" phenomenon after temporary coronary occlusion in the dog. J Clin Invest. 1974;54(6):1496-508. https://doi.org/10.1172/JCl107898.

53. Thygesen K, Mair J, Mueller C, Huber K, Weber M, Plebani M, et al. Recommendations for the use of natriuretic peptides in acute cardiac caret: a position statement from the study group on biomarkers in cardiology of the ESC working group on acute cardiac care. Eur Heart J. 2011;33(16):20016. https://doi.org/10.1093/eurheartj/ehq509.

54. Ardissino D, Merlini PA, Bauer KA, Galvani M, Ottani F, Franchi F, et al. Coagulation activation and long-term outcome in acute coronary syndromes. Blood. 2003;102(8):2731-5. https://doi.org/10.1182/blood-200203-0954. 
55. Morelli F, Schol-Gelok S, Arends LR, Boersma E, Kruip M, Versmissen J, et al. Effect of antiplatelet drugs on D-dimer levels: a systematic review and Meta-analysis. J Cardiovasc Pharmacol. 2019;73(6):343-51. https://doi.org/1 $0.1097 / F J C .0000000000000670$.

56. Ding S, Xu S, Chen X, Wu S. Effects of atorvastatin combined with bivalirudin on coagulation function, cardiac function, and inflammatory factors of percutaneous coronary intervention in elderly patients with acute myocardial infarction. Ann Palliat Med. 2020;9(4):1905-11. https://doi.org/1 0.21037/apm-20-925.

57. Oldgren J, Budaj A, Granger CB, Khder Y, Roberts J, Siegbahn A, et al. Dabigatran vs. placebo in patients with acute coronary syndromes on dual antiplatelet therapy: a randomized, double-blind, phase II trial. Eur Heart J. 2011;32(22):2781-9. https://doi.org/10.1093/eurhearti/ehr113.

58. Mega JL, Braunwald E, Wiviott SD, Bassand J-P, Bhatt DL, Bode C, et al. Rivaroxaban in patients with a recent acute coronary syndrome. N Engl J Med. 2011;366(1):9-19. https://doi.org/10.1056/NEJMoa1112277.

59. Eikelboom JW, Connolly SJ, Bosch J, Dagenais GR, Hart RG, Shestakovska O, et al. Rivaroxaban with or without aspirin in stable cardiovascular disease. N Engl J Med. 2017;377(14):1319-30. https://doi.org/10.1056/NEJMoa1709118.

60. Carvalho JF, Belo A, Congo K, Neves D, Santos AR, Piçarra B, et al. Left main and/or three-vessel disease in patients with non-ST-segment elevation myocardial infarction and low-risk GRACE score: prevalence, clinical outcomes and predictors. Rev Port Cardiol. 2018;37(11):911-9. https://doi. org/10.1016/.j.repc.2018.03.016.

61. Antoniucci D, Valenti R, Migliorini A, Moschi G, Bolognese L, Cerisano G, et al. Direct infarct artery stenting without predilation and no-reflow in patients with acute myocardial infarction. Am Heart J. 2001;142(4):684-90. https://doi.org/10.1067/mhj.2001.117778.

62. Qin T, Xie L, Chen MH. Meta-analysis of randomized controlled trials on the efficacy and safety of intracoronary administration of tirofiban for no-reflow phenomenon. BMC Cardiovasc Disord. 2013;13(1):68. https://doi.org/10.11 86/1471-2261-13-68.

63. Zeng $\mathrm{Q}$, Zhang LD, Wang W. A meta-analysis of randomized controlled trials investigating tirofiban combined with conventional drugs by intracoronary administration for no-reflow prevention. Anatol J Cardiol. 2021;25(1):7-16. https://doi.org/10.14744/AnatolJCardiol.2020.99469.

64. Rossington JA, Sol E, Masoura K, Aznaouridis K, Chelliah R, Cunnington M, et al. No-reflow phenomenon and comparison to the normal-flow population postprimary percutaneous coronary intervention for ST elevation myocardial infarction: case-control study (NORM PPCI). Open Heart. 2020; 7(2):e001215. https://doi.org/10.1136/openhrt-2019-001215.

\section{Publisher's Note}

Springer Nature remains neutral with regard to jurisdictional claims in published maps and institutional affiliations.

Ready to submit your research? Choose BMC and benefit from:

- fast, convenient online submission

- thorough peer review by experienced researchers in your field

- rapid publication on acceptance

- support for research data, including large and complex data types

- gold Open Access which fosters wider collaboration and increased citations

- maximum visibility for your research: over $100 \mathrm{M}$ website views per year

At $\mathrm{BMC}$, research is always in progress.

Learn more biomedcentral.com/submissions 